\title{
HYDROGEL MICROVALVES FABRICATED USING IN-SITU POLYMERIZATION
}

\author{
Robin H. Liu*, Qing Yu*, Joseph M. Bauer*, Jeffrey S. Moore*, David J. Beebe* ${ }^{\dagger}$ \\ *The Beckman Institute for Advanced Science and Technology \\ University of Illinois at Urbana-Champaign \\ Urbana, Illinios 61801, USA \\ ${ }^{\dagger}$ Department of Biomedical Engineering, University of Wisconsin-Madison, \\ Madison, Wisconsin 53706, USA (dbeebe@engr.wisc.edu)
}

\begin{abstract}
Hydrogel microvalves, which consist of a single smart hydrogel material that undergoes a volume change in response to changes in local $\mathrm{pH}$, have been developed. The hydrogel components were fabricated inside microchannels using in-situ photopolymerization. Two 2D shut-off valves and one 3D hybrid valve were fabricated and tested. Flow and pressure performances were studied. Analytical modeling was performed on the $3 \mathrm{D}$ valve. The behavior of hydrogel valves in electrokinetic flow was also investigated. Hydrogel microvalves have a number of advantages over conventional microvalves, including relatively simple fabrication, no external power requirement, no integrated electronics and large displacement.
\end{abstract}

Keywords: in-situ polymerization, microvalves, hydrogel

\section{INTRODUCTION}

Most conventional active microvalves couple a flexible diaphragm to a thermopneumatic, piezoelectric, electrostatic, electromagnetic, or bimetallic actuator [1]. The scaling of these actuation forces with respect to the size of the devices is often unfavorable to micro-actuators [2]. Some actuator components are made heavy and large ( $\mathrm{mm}$ size) in order to produce enough force to regulate a microscale fluid. The displacement of conventional diaphragms (e.g., silicon, silicon nitride) is often limited to tens of microns or less. Many actuators use resistive heating (thermopneumatic), high voltage (electrostatic), and bulky magnets (electromagnetic), which often require high power consumption. Moreover, the integration of many microvalves composed of electronic components into complex microfluidic systems has proven to be non-trivial using traditional approaches, such as modular or lithographic methods [3]. In general, most conventional microvalves are complex and costly, and integrating them into micro-systems is challenging

We have recently developed an in-situ liquid phase polymerization process to integrate functional materials into microfluidic channels to construct microvalves $[4,5]$. The microvalves consist of a stimuli-responsive hydrogel material that undergoes a volume change in response to changes in local $\mathrm{pH}$. Stimuli-responsive hydrogels have a significant advantage over conventional microfluidic actuators due to their ability to undergo abrupt volume changes in response to the surrounding environment without the requirement of an external power source. Stimuliresponsive hydrogels are crosslinked polymer networks that can undergo discontinuous volume changes in response to chemical signals in their surrounding environment [6]. Among stimuli- responsive hydrogels, $\mathrm{pH}$ sensitive hydrogels are one of the most commonly studied. As the environmental $\mathrm{pH}$ varies, the hydrogel networks change from neutral to charged because of the protonation of amine groups or the deprotonation of acid groups. The hydrogels thus undergo a volume transition from the collapsed state to the expanded state due to osmotic pressure exerted by mobile counter-ions neutralizing the network charges. In this paper, we report an extension of our previous work and detailed characterizations of the microvalves with optimized design.

\section{ANALYTICAL MODELING}

Among the hydrogel microvalves constructed, the $3 \mathrm{D}$ hybrid valve is the only one that couples an axisymmetric flexible poly(dimethylsiloxane) (PDMS) membrane to a hydrogel actuator. An analytical model was used to optimize the design of the $3 \mathrm{D}$ valve. The objective is to determine the relationship between the membrane deflection $(z)$, the membrane thickness $(t)$, and the diameter of the membrane $(2 R)$, for a given pressure applied by a hydrogel actuator (Fig.1). Consider a thin circular membrane of radius $R$ that is clamped along the perimeter and subjected to a net uniform pressure $p$ exerted by the hydrogel actuator. Since the deflection of the membrane $z$ (hundreds of microns) is an order of magnitude larger than its thickness $t$ (tens of microns), and the lateral deflection $w$ is more than a few tenths of thickness $t$, the "membrane" theory was employed instead of the "thin plate" theory $[7,8]$. Bending moments are assumed to be negligibly small. The membrane force that is acting tangent to the deflected middle surface due to tension is relatively large, and it is assumed to be uniform across the thickness of the membrane. The model assumes that the membrane material is isotropic and linearly elastic at small strain.

Let $T$ denote the tension per unit length of the membrane. The net force on the membrane perpendicular to a section of radius due to $p$ is $\pi r^{2} p$ (see $\mathrm{r}$ in Fig. 1). Note that $h$ in Fig. 1 is assumed to be large enough so that the membrane does not touch the orifice. The restoring force due to tension in the membrane is $2 \pi r \sin \theta$. Force equilibrium requires: $2 \pi r T \sin \theta+\pi r^{2} p=0$, where $\sin \theta=d z / d r$. The solution is given by

$$
z=-\frac{p}{4 T} r^{2}
$$

The strain along the radial direction $r$ is

$\varepsilon_{r}=\left(\int_{0}^{R} \sqrt{1+z^{\prime 2}} d r-R\right) / R \approx \frac{p^{2} R^{2}}{24 T^{2}}$

Together with Hooke's law $\varepsilon_{r}=\sigma_{r} / E^{*}$, where $E^{*}=E /\left(1-v^{2}\right)$, we have 


$$
T=\sigma_{r} t=\frac{\varepsilon_{r} E}{1-v^{2}} t=\left(\frac{1}{24} \frac{E}{1-v^{2}} t p^{2} R^{2}\right)^{1 / 3}
$$

which together with Eq. (1), gives

$$
\left.z_{\max }\right|_{r=R}=-\frac{1}{4}\left[\frac{24\left(1-v^{2}\right) p}{E}\right]^{1 / 3} \frac{R^{4 / 3}}{t^{1 / 3}}
$$

or

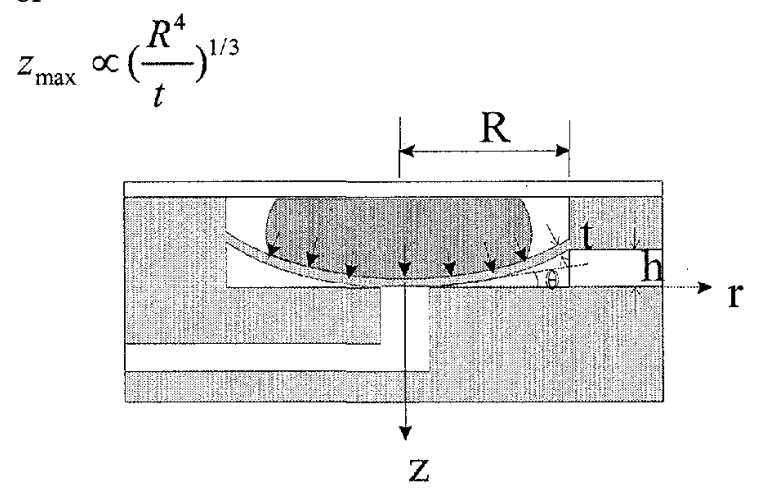

Fig. I Schematic of the 3D hybrid valve.

Fq. (2) indicates that the maximum deflection of the membrane increases only slightly when the thickness of the membrane is decreased, while a large change results from increased membrane radius. In order to obtain large $z_{\max }$, we can either make a thinner membrane or a membrane with larger diamctcr.

The pressure $p$ generated by the hydrogel actuator is unknown. But if $z_{\max }$ can be measured experimentally, then the pressure can be determined by Eq. (2):

$$
p=-\frac{8 z_{\max }^{3} E t}{3\left(1-v^{2}\right) R^{4}}
$$

\section{EXPERIMENTS}

Two 2D shut-off valves and one $3 D$ hybrid valve were fabricated. The fabrication process consists of two steps: fabrication of microchannels and in-situ polymerization. For the 2D valves, microchannels are made of EPON (Nano XP SU-8 50, Microchem Corp., Newton, MA). A transparent adhesive tape (regular packaging lape) was used to seal the $200 \mu \mathrm{m}$ deep EPON microchannels. The microchannels of the 3D valve were constructed using multi-layer PDMS [9]. A glass cover slip served as the top of the channel. After the fabrication of microchannels, an in-situ polymerization that introduces active hydrogel components into microchannels via direct photopatterning of a liquid phase was used to complete the microvalve construction. The liquid phase mixture that consists of monomers (acrylic acid and 2-hydroxyethyl methacrylate in a 1:4 mol. ratio), a crosslinker (ethylene glycol dimethacrylate, $1 \mathrm{wt} \%)$ and a photoinitiator $(2,2-$ dimethoxy phenylacetophenone, $3 \mathrm{wt} \%$ ) was first flowed into the microchannel. After the liquid reached a quiescent state in the microchannel, it was then exposed to UV light through a photomask placed on top of the channels. When the polymerization was finished, the channel was flushed with deionized (DI) water to remove unpolymerized liquid. The resulting hydrogel expands in basic solutions and collapses in acidic solutions, with the volume transition at $\mathrm{pH} 4.5 \sim 7.0$. The details of microvalve fabrication process can be found elsewhere $[4,5]$.

Pressure drop measurements using a pressure transducer (Validyne Engineering Co, Northridge, CA) with a pressure range of 0 to 125 psi were taken over the regulated channel length to characterize the hydrogel microvalves. In order to verify fluid flow in the various device designs, a variety of flow visualization techniques such as particle tracking, dyes, and air bubbles were used. To view the membrane deflection of the 3D hybrid microvalve, the cross-section images of the valve were obtained by cutting the PDMS using a razor blade without damaging the valve.

\section{RESULTS AND DISCUSSION}

A shut-off valve, consisting of a single hydrogel component that can sense the chemical environment $(\mathrm{pH})$ in one channel and regulate the flow in an adjacent channel, was constructed as shown in Fig.2. The hydrogel structure was polymerized in the gap (300 $\mu \mathrm{m}$ wide) between two parallel microchannels. The sensing channel ( $600 \mu \mathrm{m}$ wide) was constructed wider than the regulated channel ( $300 \mu \mathrm{m}$ wide) in order to keep the sensing channel open when the regulated channel is completely shut off. Solutions of different $\mathrm{pH}$ values were pumped through the sensing channel while DI water flowed through the regulated channel. As the $\mathrm{pH}$ in the sensing channel was changed from 3.8 to 5.7 , the shut-off response time of the hydrogel is slow $(\sim 160 \mathrm{~min})$ due to the slow migration of ions across the hydrogel. Cross talk between two channels is another problem that may limit some practical applications.

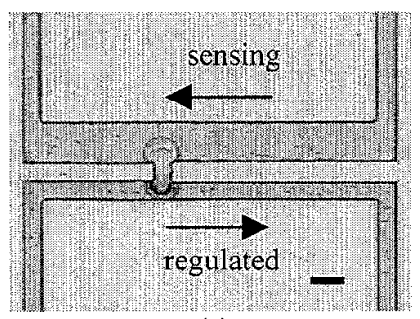

(a)

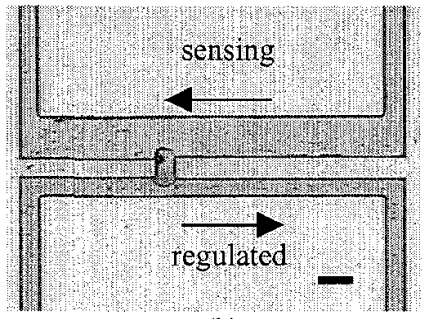

(b)
Fig. 2 Micrographs of a $2 D$ hydrogel shut-off valve. (a) and (b) illustrate flow regulation in the regulated channel as the $p H$ is changed from 3.8 to 5.7 in the sensing channel.

Since diffusion is the rate-limiting factor governing the swelling process of hydrogels [10], the time response of the volume change approximately follows the square of the hydrogel dimension. In order to improve response time, smaller hydrogels with a larger surface arca cxposed to surrounding chemical environment are preferred. However, it was found that small hydrogel objects tend to buckle or migrate during a volume change. In order to fabricate stable hydrogel actuators with fast response times, a hydrogel jacket ( $50 \mu \mathrm{m}$ thick) was polymerized around a prefabricated circular EPON post $(50 \mu \mathrm{m}$ diameter) in the microchannel (Fig.3). When a $\mathrm{pH} 11$ solution was flowed into a side branch, the hydrogel jacket expanded and closed the regulated channel ( $\mathrm{pH} \mathrm{7)}$. If a $\mathrm{pH} 2$ solution was flowed into the other side branch instead, the contracted hydrogel allowed the fluid in the regulated channel to flow down the waste channel. The post provides a robust support and also improves time response due to the short diffusion path of the hydrogel jackets surrounding the posts. The response time of the post valve design is 12 seconds 
(Fig. 4). This integration of hydrogels into microfluidic systems provides the scaling necessary to overcome the primary drawback (slow time response) of hydrogels. The maximum differential pressure the valve can sustain is 56.5 psi when the valve is closed. At least 200 operating cycles were achieved over a $\mathrm{pH}$ range of 2 to 11 without failure.

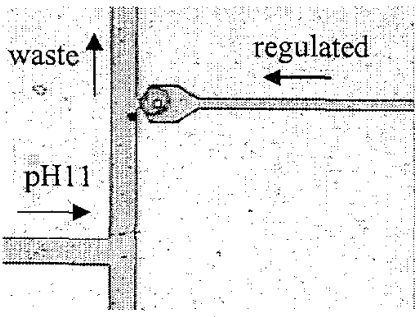

(a)

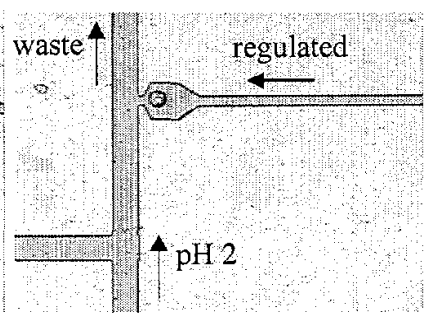

(b)
Fig. 3 (a) Micrograph (2x) of the hydrogel jackets blocking the regulated channel ( $p H$ 7) in their expanded state in a pH 11 solution. (b) Micrograph showing the contracted hydrogels allowing the fluid to flow down the side branch.

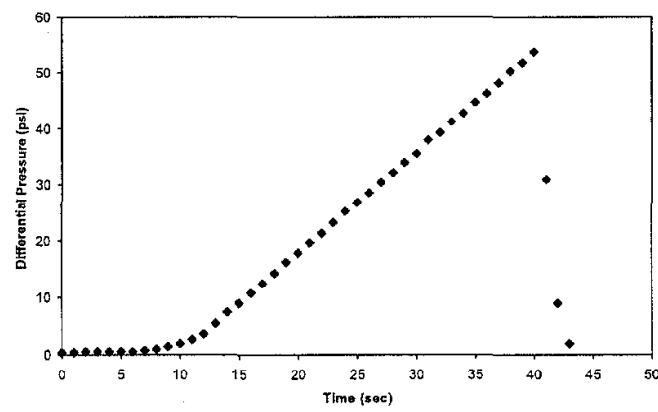

Fig. 4 Pressure drop measurement of the $2 D$ post valve illustrates the time response of the valve during swelling process. The transition to a linear response (at approx. $12 \mathrm{sec}$ ) indicates valve closure. At $41 \mathrm{sec}$, the pressure dropped substantially due to the failure of an external tubing connection.

The post hydrogel valve was also tested in electrokinetic flow (EOF) experiments. As shown in Fig. 5, the regulated channel was first filled with a buffer solution ( $\mathrm{pH} 7$ ) using a syringe pump. The hydrogel jacket expanded and closed the channel. A voltage of 500 $\checkmark$ was then applied to the ends of the channel. The electrical field resulted in a rapid additional expansion $(\sim 2 \mathrm{sec})$ of the hydrogel jackct. The resulting force was large cnough that it dclaminated the EPON post from the channel bottom. Although this phenomenon is still being investigated, the preliminary result demonstrates a potential use of hydrogel components as actuators in EOF systems.

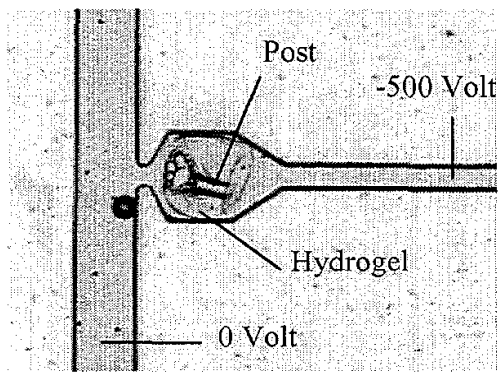

Fig.5 Micrograph (4x) illustrates a post hydrogel microvalve in $E O F$.
Since hydrogels are generally porous, ions can diffuse through a hydrogel object, resulting in a leaking or cross talk problem in the above $2 \mathrm{D}$ hydrogel valves. To address this problem, the 3D hybrid microvalve couples a flexible PDMS membrane to a hydrogel actuator (Fig.6). The membrane acts to physically separate the sensing and regulated flows. The hydrogel actuator can sense the $\mathrm{pH}$ in the upper channel and expands or contracts as the surrounding $\mathrm{pH}$ is changed. The force associated with these volumetric changes is sufficient to deform the membrane and consequently control the flow in the lower channel. The membrane can deform to completely block the orifice with a displacement of up to $150 \mu \mathrm{m}$. No leakage was observed. We previously reported a maximum differential pressure $P_{\max }$ of 7.5 psi if the lower channel height $h$ is $150 \mu \mathrm{m}$ [5]. If $h$ decreases while other parameters are kept unchanged (i.e., the diameter of the hydrogel actuator is 250 $\mu \mathrm{m}$, the PDMS membrane has a thickness of $50 \mu \mathrm{m}$ and a $700 \mu \mathrm{m}$ diameter), $P_{\max }$ goes up as shown in Table 1 . In other words, $h$ should be kept as small as possible in order to achieve high $P_{\max }$. However, it was found that if $h$ is less than $70 \mu \mathrm{m}$, the PDMS membrane is prone to stick to the bottom of the lower channel due to a surface force during the PDMS bonding process, rendering the orifice sealed permanently. Therefore, it is recommended that the ratio of $h$ to the diameter of the membrane be at least 0.1 if high $P_{\max }$ is required. The maximum differential pressure $P_{\max }$ was measured to be 26.7 psi with a height $h$ of $75 \mu \mathrm{m}$ (Fig. 6).

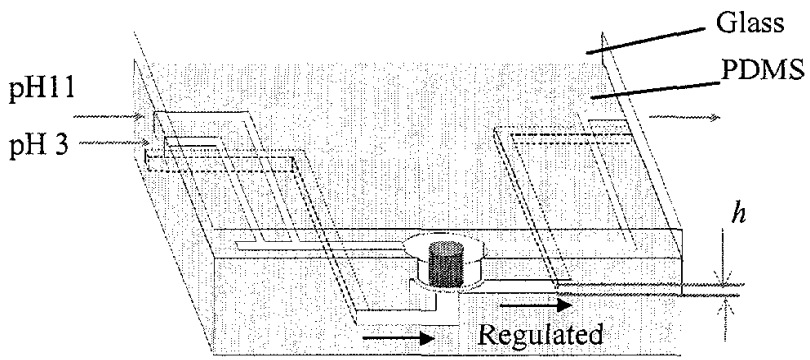

(a)

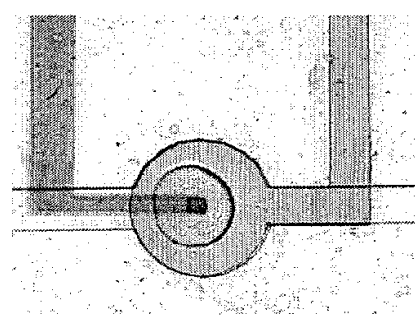

(b)

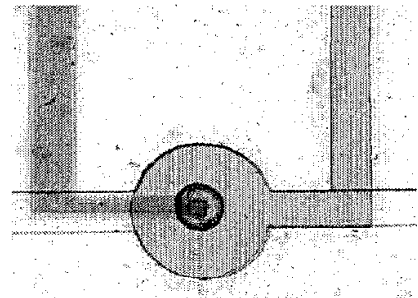

(d)
Hydrogel

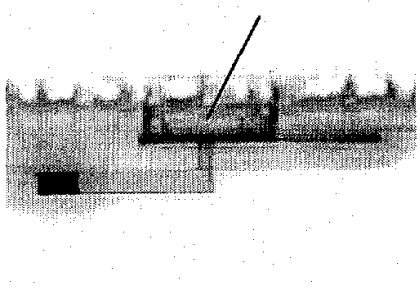

(c)

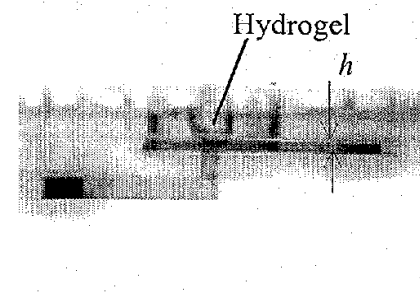

(e)
Fig. 6 (a) Schematic of a $3 D$ hybrid microvalve. (b), (c), The top view and the cross section, respectively, of the valve when a $\mathrm{pH} 11$ solution is flowing in the upper channel. The hydrogel actuator expands and deforms the PDMS membrane to seal the orifice in the regulated channel. (d), (e) show that the hydrogel contracts at a pH 3 solution and reopens the regulated channel. 
Table 1 The effect of the lower channel height $h$ on the maximum differential pressure $P_{\text {max }}$ of the $3 D$ hybrid microvalve.

\begin{tabular}{|c|c|}
\hline $\boldsymbol{h}$ ( $\boldsymbol{\mu m})$ & $\boldsymbol{P}_{\max }(\boldsymbol{p s i})$ \\
\hline 75 & 26.7 \\
\hline 100 & 18.4 \\
\hline 125 & 11.3 \\
\hline 150 & 7.5 \\
\hline
\end{tabular}

Moreover, small $h$ gives rise to rapid closure of the orifice. The shut-off time response of the 3D hybrid valve with $h$ of $75 \mu \mathrm{m}$ is $19 \mathrm{sec}$, compared to $50 \mathrm{sec}$ if $h$ is $150 \mu \mathrm{m}$. At least 200 operating cycles were achieved over a $\mathrm{pH}$ range of 3 to 11 without failure.

From the experiment, it was found that $z_{\max }$ is $185 \mu \mathrm{m}$ for a hydrogel actuator $(250 \mu \mathrm{m}$ diameter and $200 \mu \mathrm{m}$ high $)$ at $\mathrm{pH} 11$ deforming a PDMS membrane of $50 \mu \mathrm{m}$ thickness and $700 \mu \mathrm{m}$ diameter (note: the PDMS membrane does not touch the orifice because $h$ is $200 \mu \mathrm{m}$ here). Therefore, Eq.(3) is used to determine the pressure generated by the hydrogel actuator. Given that the Young's modulus $E$ of PDMS (1:10 mixing ratio) is $750 \mathrm{kPa}$ and the Poisson ratio is 0.5 [11], the pressure $p$ is approximately 56.26 $\mathrm{kPa}$, which corresponds to a force of $22 \mathrm{mN}$.

Due to the unique ability to convert chemical energy into mechanical work, the pH-sensitive hydrogels exhibit both sensing and actuating functions simultaneously. This approach avoids the need for integrated electrical connections for microvalve operation. Moreover, the fabrication process of hydrogel microvalves is less complicated than that of most conventional microvalves which involve various bulk processes (e.g., bulk etching of Si wafers) and surface processes (e.g., thin film processes). This method avoids the complicated integration process required to construct conventional microvalves inside complex microfluidic systems. Although the time response of the hydrogel microvalves shown above is relatively slow compared to most conventional microvalve ( $\sim \mathrm{ms})$, it is believed that changes in hydrogel chemistry and valve design can further improve the time response and make hydrogel actuation widely applicable to microfluidic systems.

\section{CONCLUSION}

Two 2D shut-off hydrogel microvalves and one 3D hybrid microvalve have been fabricated using an in-situ polymerization. The time response $(\sim \mathrm{sec})$ of hydrogel actuators has been improved by scaling down the hydrogel objects. The maximum differential pressure was 56.5 psi for the $2 \mathrm{D}$ valve and $26.7 \mathrm{psi}$ for the $3 \mathrm{D}$ valve. Simple fabrication and no power consumption or integrated electronics make hydrogel microvalves attractive in many microfluidic applications.

\section{ACKNOWLEDGEMENT}

The authors would like to express their thanks to Kendra Sharp at UIUC for her assistance in pressure measurement, Dr. Byung-Ho Jo at UIUC for his help in PDMS fabrication, Dr. Alex Sassi at Aclara Biosciences Inc., Prof. Taher Saif and Prof. Ronald Adrian at UIUC for useful discussions. This work has been supported under a grant from DARPA-MTO (\# F33615-98-12853) (Program manager: Dr. Abraham Lee).

\section{REFERENCES}

1. G. T. A. Kovacs, Micromachined Transducers Sourcebook, New York: McGraw-Hill, 1998.

2. W. S. N. Trimmer, "Microrobots and micromechanical systems," Sensors and Actuators, vol. 19, pp. 267-287, 1988.

3. M. Madou, Fundamentals of Microfabrication. Boca Raton, FL: CRC Press LLC, 1997.

4. D. J. Beebe, J. S. Moore, J. M. Bauer, Q. Yu, R. H. Liu, C. Devadoss, B.-H. Jo, "Functional Structures For Autonomous Flow Control Inside Microfluidic Channels", Nature, in press, 2000.

5. R. H. Liu, Q. Yu, J. M. Bauer, B.-H. Jo, J. S. Moore, D. J. Beebe, "In-channel Processing to Create Autonomous Hydrogel Microvalves", accepted in $\mu T A S$ 2000, Netherlands, 2000.

6. Y. Osada and S.B.Ross-Murphy, "Intelligent Gels," Scientific American, pp.82-87, 1993.

7. R. D. Cook and W. C. Young, Advanced Mechanics of Materials, New York: Macmillan, 1994.

8. M. T. A. Saif, B. E. Alaca, and H. Sehitoglu, "Analytical Modeling of Electrostatic Membrane Actuator for Micro Pumps", IEEE J. of Microelectromechanical Systems, Vol. 8, No. 3, Sept, 1999 , pp. 335-345.

9. B. H. Jo, L. M. Van Lerberghe, K. M. Motsegood and D. J. Beebe, "Three-dimensional Micro-channel Fabrication in Polydimethylsiloxane(PDMS) Elastomer", Journal of Microelectro-mechanical Systems, Vol. 9, No. 1, March, 2000, pp. 7681.

10. E. S. Matsuo and T. Tanaka, "Kinetics of Discontinuous Volume-Phase Transition of Gels," J. Chem. Phys., vol. 89, pp. 1695-1703, 1988.

11. D. Armani and C. Liu, "Re-configurable Fluid Circuits By PDMS Elastomer Micromachining", 12th International Conference on MEMS, MEMS 99, Orland, FL, 1998, pp.222-227. 